\title{
Service Robots in Wuhan Cabin Hospitals
}

\author{
Sheng Bao, ${ }^{\# \#}$ Bill Huang, ${ }^{2 \#}$ Jianjun Yuan, ${ }^{1 *}$ Bing Wang, ${ }^{2 * *}$ \\ Lin Xia, ${ }^{2}$ Mingyuan Wang, ${ }^{1}$ and Yanchen $\mathrm{Liu}^{2}$ \\ ${ }^{1}$ Shanghai Robotics Institute, Shanghai Key Laboratory of Intelligent Manufacturing and Robotics, \\ School of Mechatronic Engineering and Automation, Shanghai University, \\ 99 Shangda Road, Baoshan District, Shanghai 200444, China \\ ${ }^{2}$ CloudMinds Technologies Inc., 85 Hong'an Road, Fangshan District, Beijing 102488, China
}

(Received December 25, 2020; accepted May 25, 2021)

Keywords: medical service robot, human-robot interaction, COVID-19, sensor fusion

The cabin hospitals used for COVID-19 victims have an infectious environment and thus require highly autonomous systems to provide various services. This article presents a multirobot architecture designed for cabin hospitals with special focus on the sensors used by service robots. The robots' functions include autonomous disinfection, delivery, cleaning, temperature measurement, physical interaction, and conversation assistance. The robots collaborate with each other by using multiple sensors in large and complicated scenes and evolve together. The robots are controlled by cloud controllers considering a human-centered multi-robot collaboration mechanism. This article presents the details of these robots, including the hardware and software architecture of the robots with sensors, the communication modules, and the cloudbased central controllers. It also presents several applications of the robots and discusses open problems.

\section{Introduction}

The cabin hospitals in Wuhan were special hospitals built to accept COVID-19-infected patients with mild symptoms ${ }^{(1,2)}$ to avoid pre-symptomatic, symptomatic, or asymptomatic transmission. ${ }^{(3)}$ The hospitals were opened on February 5th, 2020 as temporary low-cost infectious disease hospitals, similarly to battlefield hospitals. Images of a cabin hospital are shown in Fig. 1. To quickly deploy and respond to the epidemic, the cabin hospitals require a contact-free environment, and there is a strong need for robotic assistance to reduce crossinfection. ${ }^{(4,5)}$ Under this background, we developed a cloud robotic architecture for cabin hospitals.

The cabin hospitals have the following features and requirements for robots:

- The indoor ground is generally flat, the walkways and compartments usually have sufficient moving space for people and robots, and the indoor light is sufficient. Mobile robots can easily move around to complete repetitive tasks.

\footnotetext{
*Corresponding author: e-mail: terabit@shu.edu.cn

${ }^{* *}$ Corresponding author: e-mail: charly@cloudminds.com

${ }^{\#}$ Co-first authors.

https://doi.org/10.18494/SAM.2021.3235
}

ISSN 0914-4935 (C) MYU K.K. https://myukk.org/ 


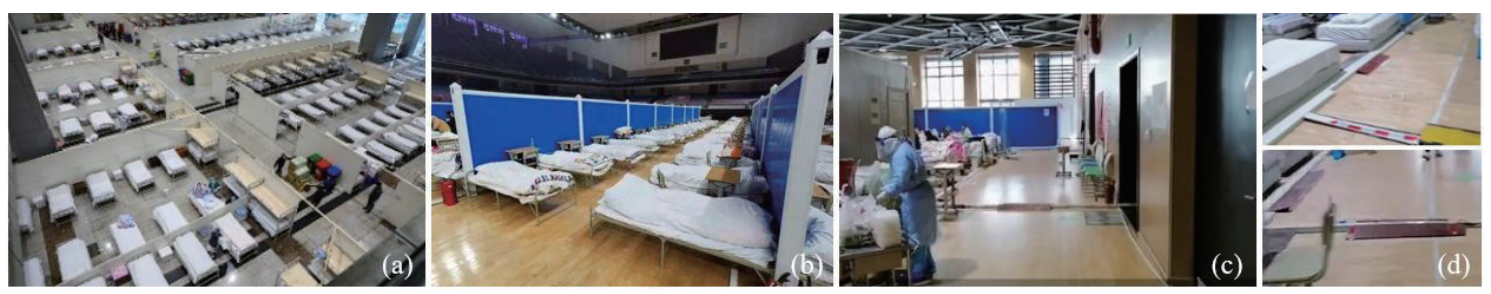

Fig. 1. (Color online) Images of a Wuhan cabin hospital. (a) Overall layout of beds. (b) Close-up view of one walkway. (c) Ground and obstacles. (d) Close-up of some details in (c).

- The environment is semi-structured and semi-open, which is suitable for pre-constructing maps. Robots may share common pre-constructed 3D maps while being corrected using multi-sensor information collected online.

- Patients and medical staff move around the working area. Robots must avoid them autonomously using the sensors inside and around the robots.

- The inside areas of cabin hospitals are covered by 5G wireless communication networks. Problems such as bandwidth, time delay, signal strength, and communication stability can thus be ignored.

Robots have long been required to take on dangerous, dull, and dirty jobs, often in environments unsuitable for humans. In 2021, Gao et al. ${ }^{(6)}$ reviewed the fundamental requirements for robotics for infectious disease management and how robotic technologies can be used in different medical scenarios. In 2009, Ozkil et al. ${ }^{(7)}$ emphasized that there are numerous tasks in hospitals where material delivery is required. Service robots include mobile platforms with control pads so that patients can be examined remotely by doctors. One of the most well-known receptionist robots named Pepper can handle visitors continuously without becoming tired, and the main function of Pepper is to direct the patients to the physician of their choice. ${ }^{\left({ }^{8}\right)}$ As part of research on human-robot interaction, in 2021, Hwang et al. ${ }^{(9)}$ proposed a social robot system using an end-to-end dialogue system in the context of hospital receptionist robots to optimize the dialogue system. Disinfection robots are widely used to spray antiseptic disinfectants over large areas. These robots are usually remotely controlled to avoid hazardous contact between the disinfectant and humans. For instance, the Light Strike Germ-Zapping Robot $^{(10)}$ is operated by trained hospital environmental services staff, while Courtney and Royall $^{(11)}$ proposed a robot that relies on simultaneous localization and mapping (SLAM) to autonomously scan and navigate a specific facility such as a laboratory. To address the two scenarios of logistics and disinfection, Tamantini et al. ${ }^{(12)}$ proposed a functional integration robotic system and carried out a two-month experiment.

The main purpose of medical service robots is to minimize human-to-human contact and to support the hospital through cleaning, disinfection, and so forth. However, the above robots were all developed under the condition of independent operation, so the data about the infectious environment and navigation routes can only be stored and managed separately. Robot communities play an important role in assisting the control of epidemics, ${ }^{(13)}$ making it valuable to study the collaboration between different types of robots and humans. 
The cloud can offer various advantages and provide good ideas for solving the existing problems of service robots. Using a series of mobile service robots with multiple sensors under central cloud control ${ }^{(14)}$ to work with many humans satisfies the above requirements. We specially focus on the sensors used by service robots as part of a human-centered ${ }^{(15)}$ multi- $^{-}$ robot $^{(16)}$ collaboration mechanism, which includes a human-robot collaborative security check, high modularization, rapid deployment under special circumstances, and strong adaptability to complicated scenarios. The functions of the involved robots include autonomous disinfection, delivery, cleaning, temperature measurement, and natural communication. The robots are not independent and are connected to each other by modern communication modules and cloudbased central controllers. They collaboratively work in large and complicated scenes and evolve together. This article presents the details of these robots with sensors, including the hardware and software architecture, the communication modules, the cloud-based central controllers, and so forth. It also presents several useful applications with robots and discusses open problems.

\section{Architecture of Medical Service Robots under a Cloud Controller}

Figure 2 shows an overview of the proposed architecture, including the series of service robots, sensors, central controllers, and their communications. The interactions between humans, the environment, and the robots produce important knowledge data. These data are captured by sensors and are uploaded to cloud controllers through the information transmission channel formed by a wireless network. The cloud controllers are responsible for data analysis and processing, decision-making, and the generation of specific execution instructions according to application requirements. The cloud controllers run on remote servers or computer clusters according to specific application conditions. In the application layer, the robots use the experiences and knowledge of the cloud to overcome the limitations of each individual's local knowledge. They share and expand the knowledge based on the cloud center, giving them a better perception of the surrounding environment and making it easier for them to react to specific applications.

The various types of robots included in the architecture are shown in Table 1. They were used to solve the problems of cleaning disinfection, measuring body temperature, reception, and delivery. The key technical issues of each type are listed in the lower part of the table. Technologies such as autonomous navigation, semantic recognition, and IR human body recognition are required to solve these issues. These robots, sensors, and the working environment form an ecosystem. They collaborate with each other and evolve together. Details of the implementation of the technologies will be presented in the following section.

\section{Essential Techniques for Service Robots}

Medical service robots are realized through the integration and fusion of various technologies. The most useful and basic functions are SLAM and navigation, semantic recognition, and IR human recognition. 


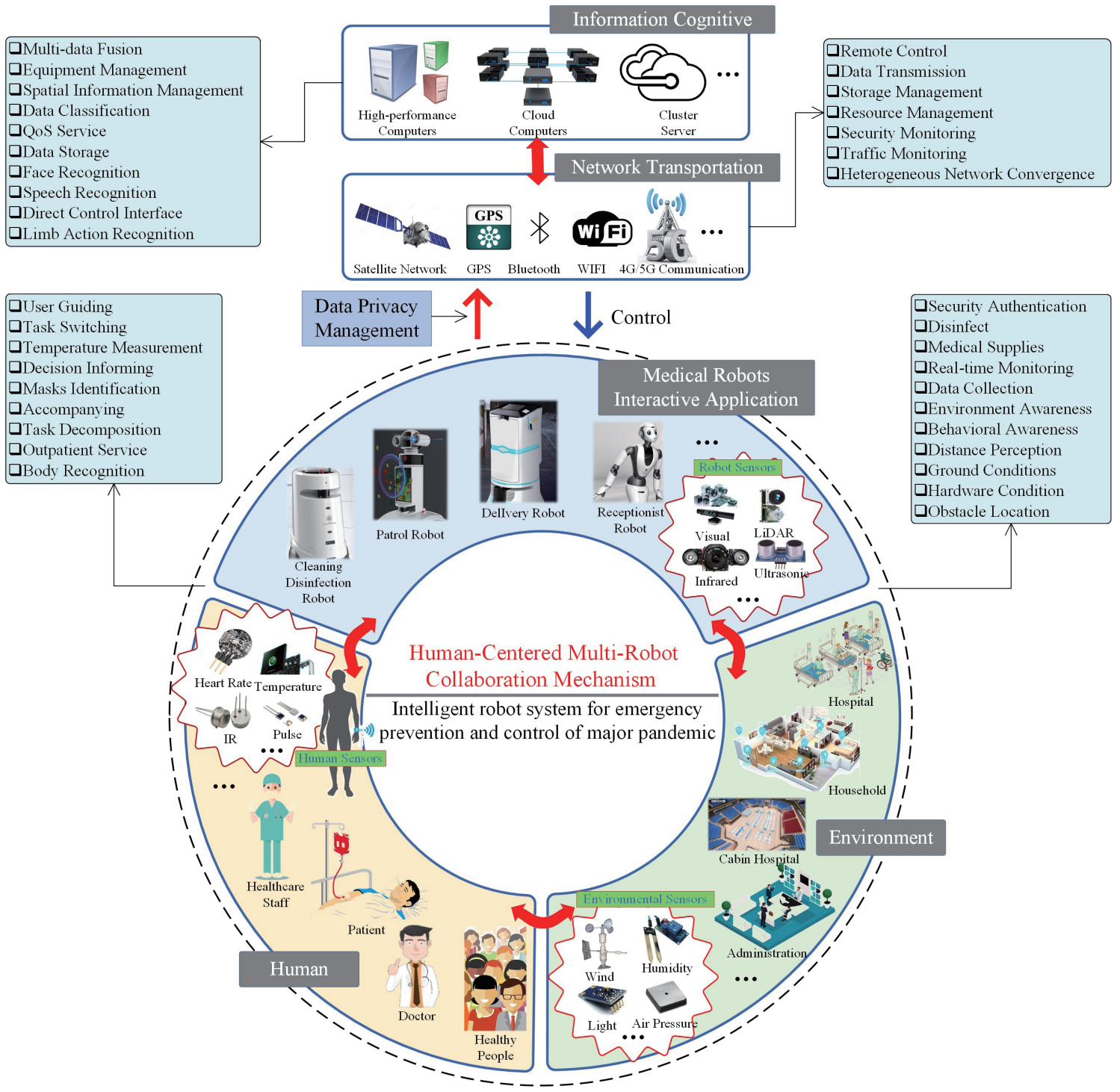

Fig. 2. (Color online) Cloud robotic architecture designed for cabin hospitals.

Table 1

Various robots involved in the architecture and their technical issues.

\begin{tabular}{lcccc}
\hline Robot Type & Cleaning/Disinfection & Patrol Security & Delivery & Reception \\
\hline & SLAM and Navigation & SLAM and Navigation & SLAM and Navigation & $\begin{array}{c}\text { Dynamic Human Body } \\
\text { Recognition }\end{array}$ \\
\cline { 2 - 5 } Technical & UV/UV-C Disinfection & $\begin{array}{c}\text { Autonomous Obstacle } \\
\text { Avoidance }\end{array}$ & $\begin{array}{c}\text { Environmental } \\
\text { Perception }\end{array}$ & $\begin{array}{c}\text { Automatic Speech } \\
\text { Recognition (ASR) }\end{array}$ \\
\cline { 2 - 5 } & $\begin{array}{c}\text { Photoionic Catalytic } \\
\text { Purification }\end{array}$ & Face Recognition & $\begin{array}{c}\text { Autonomous Obstacle } \\
\text { Avoidance }\end{array}$ & Intelligent Diagnosis \\
\cline { 2 - 5 } & $\begin{array}{c}\text { Hydrogen Peroxide } \\
\text { Vapor }\end{array}$ & IoT Technologies & Face Recognition & $\begin{array}{c}\text { Limb Movement } \\
\text { Recognition }\end{array}$ \\
\cline { 2 - 4 } & IR Thermal Imaging & & Face Recognition \\
\hline
\end{tabular}




\subsection{SLAM and navigation}

SLAM $^{(17)}$ and path planning ${ }^{(18)}$ are essential techniques to implement autonomous navigation. The cloud platform acts as an agent and service provider for robots. The cloud collects data from the robot ecosystem and connects the robot ecosystem to the computing and storage clusters through the robot operating system (ROS) and the Hadoop Distributed File System (HDFS). The server serves as a master node that maintains the ROS publisher list. The ROS nodes on the individual robot query the master node to subscribe and receive data from the HDFS or from other robots, as shown in the right of Fig. 3.

Different from the current single-robot SLAM process, ${ }^{(19,20)}$ which establishes a map through the autonomous movement of the robot, realizes its position, plans a reasonable path according to the established task, and finally executes the movement, the cloud-based architecture coordinates the robots and results through a remote server as follows:

(1) Structured maps of cabin hospitals are prepared in the cloud in advance to save time and labor. On the basis of this structured map, the robot completes the unstructured areas through dynamic scanning and continuous correction.

(2) Each robot can obtain a local map by incrementally navigating to different locations. After each robot completes its local map, the map data are uploaded to the remote servers and are integrated to complete a whole image.

(3) Each robot only performs pre-determined tasks. They no longer require a huge computing load, which helps reduce the difficulty of algorithmic design and the power consumption of individual robots, improving the system's robustness. ${ }^{(21,22)}$

We design and control each robot, such as the patrol robot shown in Fig. 3, as follows.

(1) Hardware: The following sensors are installed on the robot body, which work together to collect data: thermal imaging cameras for human IR body temperature measurement, foureye panoramic night vision devices to help patrol robots work in the dark, and sonar sensors

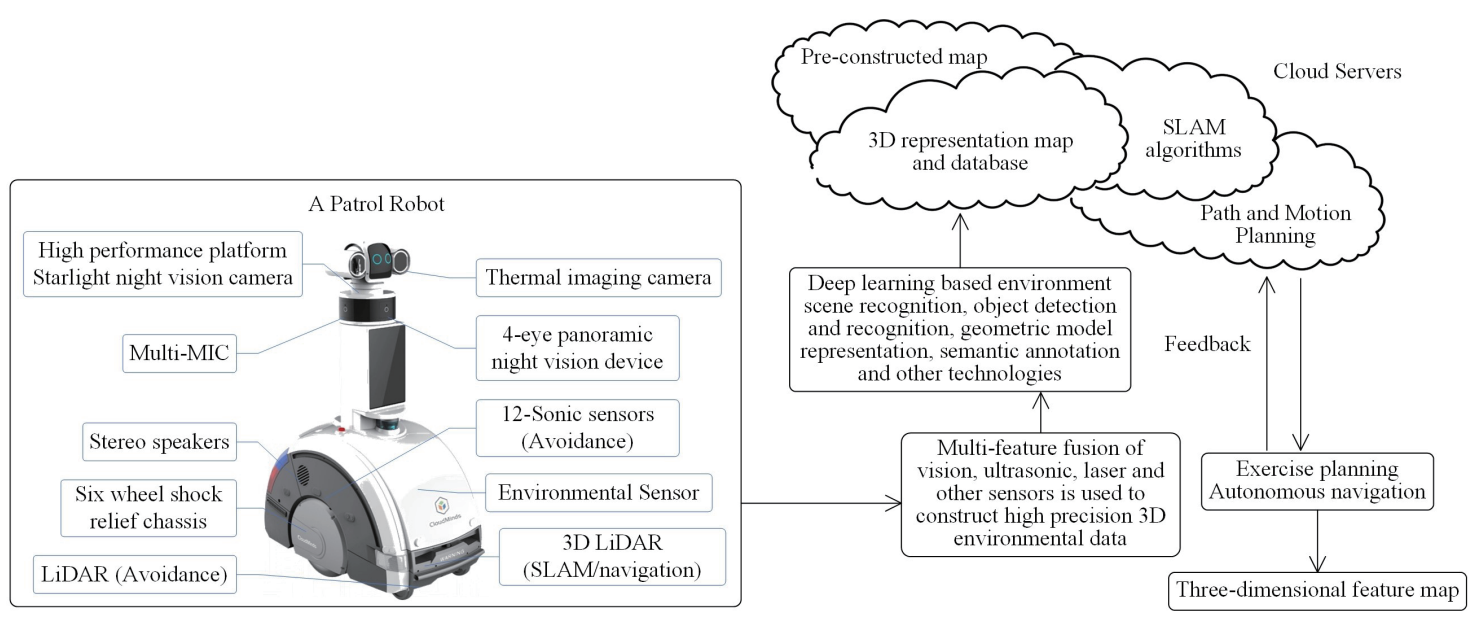

Fig. 3. (Color online) A patrol robot communicates with the cloud server to upload data and receive commands. The proposed architecture involves a series of robots that evolve together under the control of cloud servers. 
and 2D/3D cameras for SLAM and obstacle avoidance, face recognition, limb motion recognition, and so forth.

(2) 3D map construction: We construct a 3D environmental map by semantically fusing the data of robot vision, ultrasound, LiDAR, and other sensors, as shown in Fig. 4. The underlying idea is to combine deep-learning-based environmental scene recognition, object detection and recognition, geometric model representation, semantic annotation, and other technologies in the cloud. A hierarchical method is implemented. The bottom layer is the parameter layer of the sensors, which stores the parameter information of the sensors (LiDAR, camera, GPS, etc.) needed for robot positioning. The upper layer is the sensors' data layer, where the data of each sensor in the process of robot mapping and positioning are aligned and stored. The layer above that is the sparse data layer, which stores sparse 3D maps obtained by either LiDAR or cameras. Then, there is a layer for storing dense 3D maps, including dense point cloud and grid information. This layer is also used for visualization and semantic analysis. The top layer is the semantic layer, where the data are processed by detection and segmentation of the dense layer, assisted by semantic analysis. This layer represents the semantics of the 3D map and is used to construct a complete 3D representation map database.

(3) Control and planning: Along with the 3D map construction, we use the software stack with the help of ROS for planning and autonomous navigation. A complete software stack consists of both open-source and internally developed software.

\subsection{Semantic recognition}

Reception robots are required to understand patient consultation semantics and carry out communication $^{(23)}$ by analyzing and understanding emotions. They also respond to requests from patients. Implementing these functions in a single robot is difficult due to the limitations of hardware and intellectual development. Generating a personalized dialogue system requires a huge amount of storage and calculation. In contrast, the cloud server combines the multi-robot ontology to provide more humanized services to users. Its intelligent customer service system, which incorporates natural language processing (NLP) technology, recognizes the information

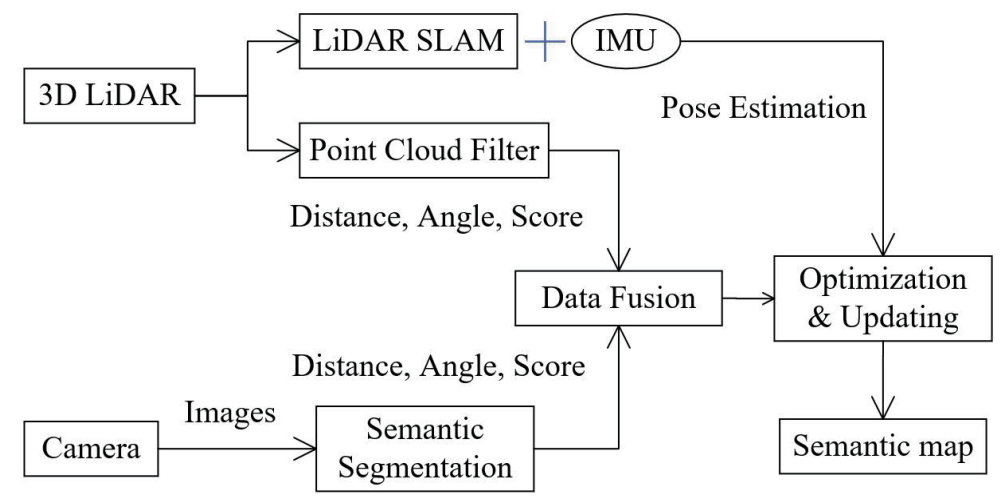

Fig. 4. Flowchart of the data fusion of 3D LiDAR and cameras of autonomous mobile robots in SLAM. 
communicated by the user and the user's emotions through patient inquiry, appointment, consultation, and so forth, and then helps, guides, and persuades the user.

The system can also predict the possible future development and spread of the epidemic through data analysis, give recommendations for prevention and control, and then help implement these measures. With the development of the epidemic, the service function requirements of the robots may become more complex. In such a case, the reception robots can participate in the work by collaborating with other robots.

We demonstrate semantic recognition by using a reception robot named Ginger as an example as follows.

(1) Hardware: Ginger is built on a platform with three omnidirectional wheels. The platform has a LiDAR sensor for mapping and navigation, sonar sensors and a 3D camera for obstacle avoidance, a 2D camera for searching for the charging connector, a 2D camera for face recognition, a 3D camera for limb motion recognition, and other auxiliary sensors such as a microphone array.

(2) ASR, NLP, and text to speech (TTS): The cloud servers run ASR, ${ }^{(24-26)} \mathrm{NLP}^{(27-29)}$ and $\operatorname{TTS}^{(30,31)}$ algorithms to perform natural communication. Figure 5 shows the relations between these algorithms. Here, ASR is used to recognize human speech and output it as text by analyzing the phoneme features of the audio signal. NLP classifies the converted text, judges the domain of the questions or commands, and returns the answers. A library of dialogue Q\&A is maintained during the NLP process using Artificial Intelligence Marker Language (AIML). The library enables multi-round judgment and counter-question management. TTS is used for speech synthesis. Text is transformed into a natural speech flow assisted by the neural network in TTS. ${ }^{(32)}$ TTS also makes the output voice smooth and rhythmically natural by leveraging some ensuing algorithms and performing directional optimization.

\subsection{Human IR recognition}

There are two main functions of human IR recognition: human body temperature measurement and mask recognition. These two functions are of equal importance for pandemic prevention in regions with high traffic.

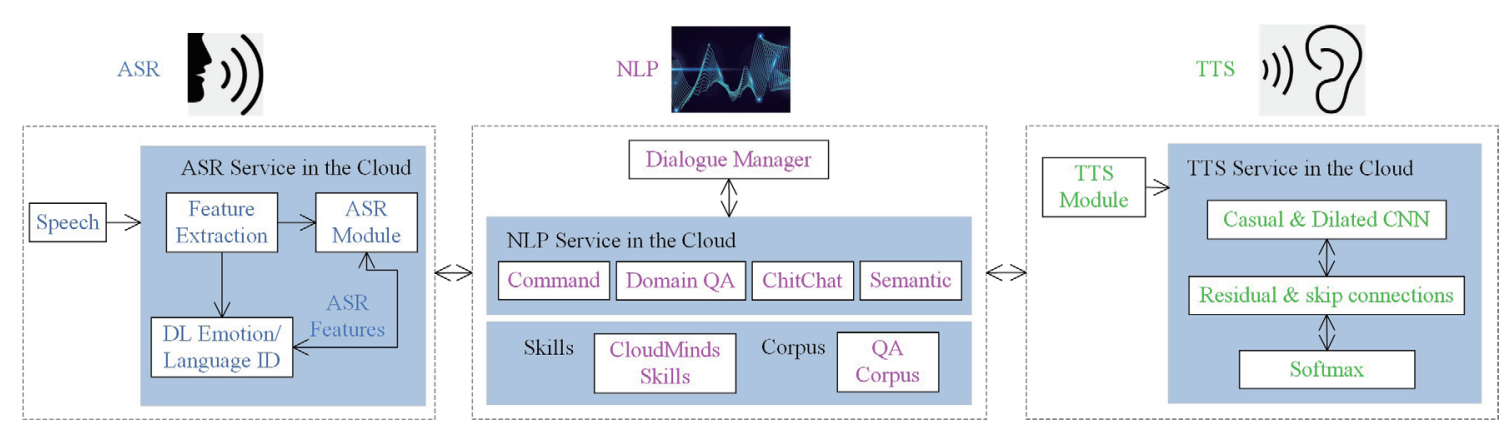

Fig. 5. (Color online) Cloud services for understanding and responding to natural language. 
(1) Continuous body temperature measurement: The temperature-measuring robots in the proposed architecture implement a contact-free body temperature measurement method to avoid cross-infection and reduce the measurement time. IR sensors ${ }^{(33)}$ are the main sensors used in the system. The essential algorithms include face detection and target tracking using thermal images and neural networks, as shown in Fig. 6. Immediately after the thermal detection of a face, the face is automatically tracked and the temperature of the region of interest (ROI) is continuously measured. The temperature-measuring robots receive and store human body temperature data through their IR sensors locally, and at the same time upload the data to the cloud servers. They can also obtain data from the cloud servers to view the changes in body temperature in a certain period.

(2) Face detection and mask recognition: The face detection algorithm is based on feature fusion in convolutional neural networks. ${ }^{(34)}$ It uses a feature fusion pyramid structure to fuse high and low layers simultaneously by spatial and channel attention learning, which prevents the semantic information on the high-level feature map from obscuring the detailed information on the low-level feature map. The spatial and channel attention also makes the semantics and details complement each other. The semantic information is used as contextual clues to improve the low-level features without losing details. ${ }^{(35)}$ The mask recognition algorithm expands the detected face area according to the proportions of the face, cuts out a sub-image of the face area, and classifies the sub-image of the face area to establish whether a mask is being worn.

Off-line training work

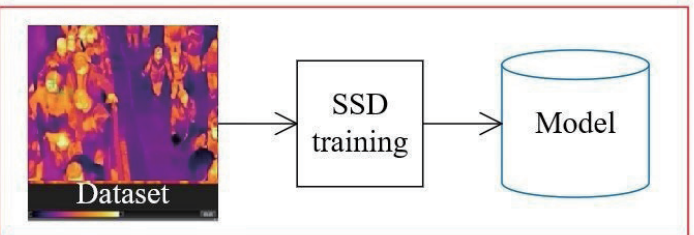

Real-time system workflow

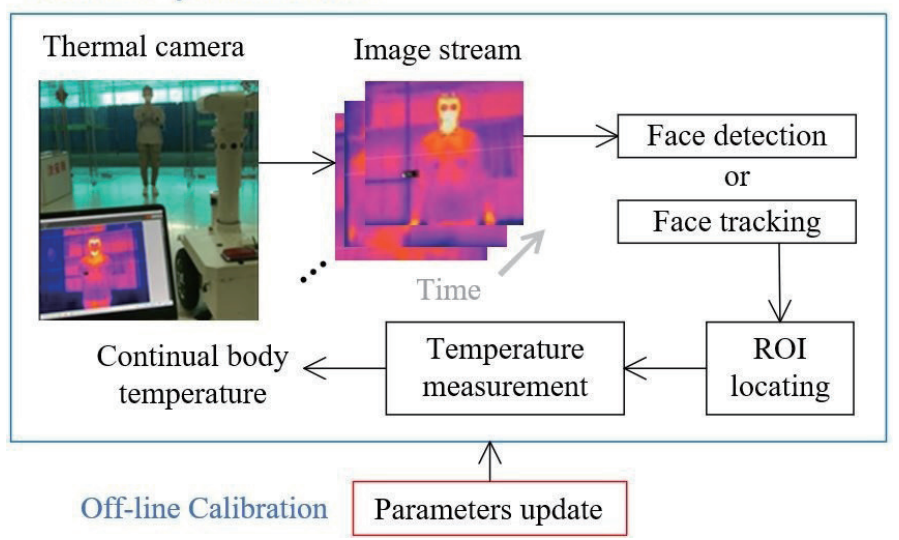

Fig. 6. (Color online) Continuous body temperature measurement by detecting and tracking human faces. 
In addition to the temperature measurement, face detection, and mask recognition, the human IR recognition module can also be quickly expanded to the safety testing of medical personnel protection equipment (i.e., whether protective clothing is being correctly worn) and to the recognition and assistance of care recipients (such as pregnant, elderly, and disabled people and children) according to specific conditions and needs.

\section{Examples and Results}

The cabin hospitals in Wuhan were temporarily built hospitals to host COVID-19 victims. The hospitals had an infectious environment and thus required highly autonomous systems to provide various services. A series of robots worked together at several cabin hospitals in Wuhan. This section shows some examples of robot operation, mainly at the cabin hospitals, such as the one built in the Hongshan Gymnasium, Wuhan. Figure 7(a) shows a logistics robot transporting medicines in a cabin hospital. Figure 7(b) shows a disinfection robot disinfecting the workspace. Figure 7(c) shows a Ginger reception robot in operation, which was designed with features including a friendly face and big eyes to help patients relax. These robots worked together to update cloud servers and receive commands. Figure 7(d) shows a view of the user interface for the cloud servers.

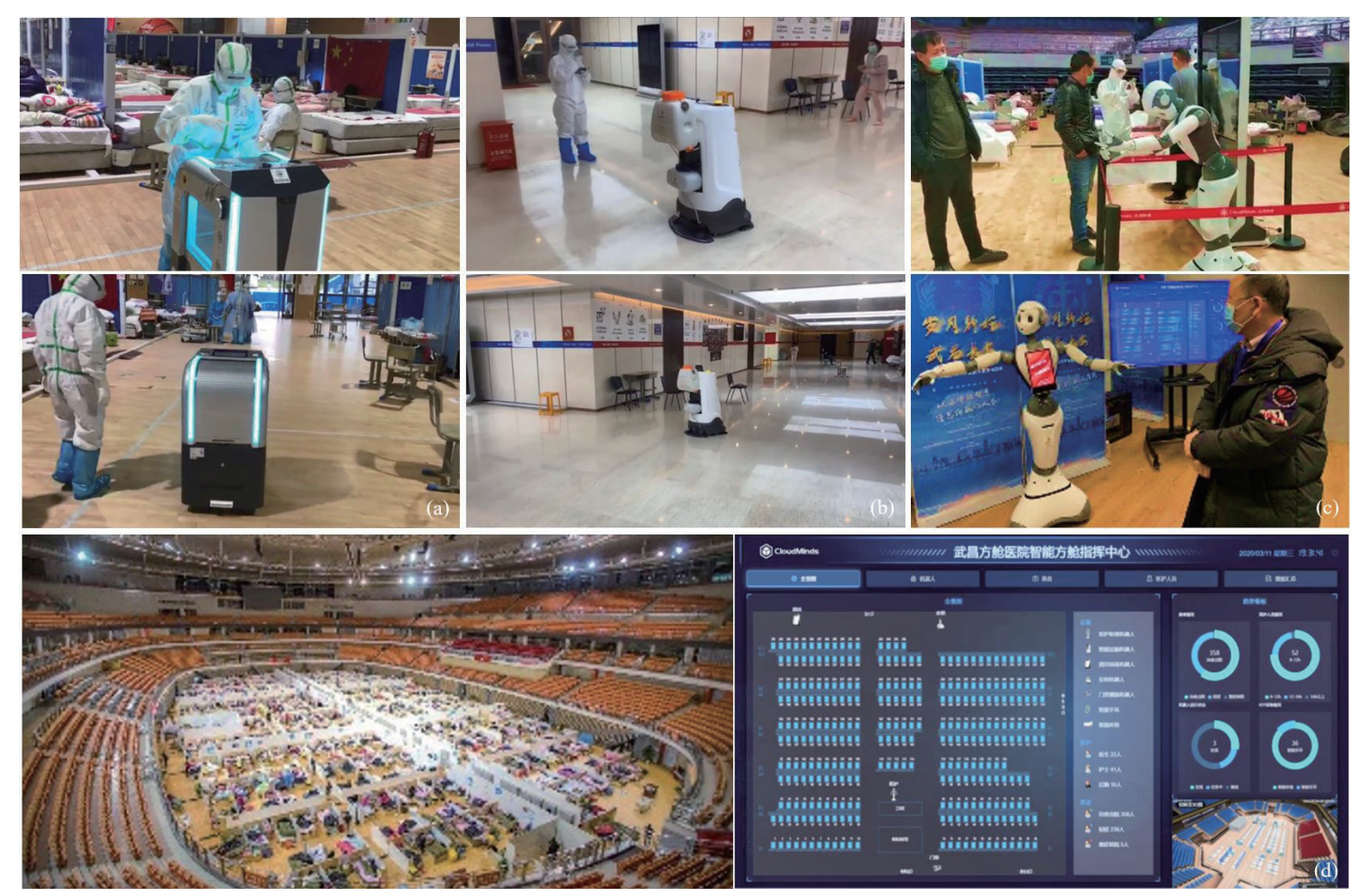

Fig. 7. (Color online) (a) Examples of human-robot interaction between hospital staff and a delivery robot. (b) Examples of cleaning and disinfection robot at work. (c) Ginger reception robot in a Wuhan cabin hospital. (d) Hospital and user interface of the cloud servers of the hospital. 
From February 2020, 21 Ginger reception robots were used in the Wuhan cabin hospitals. On March 10, all the cabin hospitals were closed and the Ginger robots were transferred to other hospitals. The numbers of times the Ginger robots communicated with people and the percentages of different topics discussed are shown in Table 2.

Figure 8 shows results obtained from the temperature measurement system and the mask recognition system at a hospital entrance. The mask recognition system locates the face area in the image, analyzes the face attributes and determines whether a mask is being worn. The same temperature measurement system was used in the Wuhan cabin hospitals and many other hospitals specially allocated to COVID-19. Table 3 lists the statistical data of temperature detection in other hospitals in Beijing, Inner Mongolia, and Anhui. The time period was from April to June 2020 and the data unit is person-times.

Table 2

Dialogue data of the Ginger reception robots.

\begin{tabular}{|c|c|c|c|}
\hline \multicolumn{2}{|c|}{ Dialogue times distribution } & \multicolumn{2}{|c|}{ Dialogue contents } \\
\hline Data & Dialogue (times) & Content & Percentage $(\%)$ \\
\hline Feb. 2020 & 11935 & Easy Chat & 37 \\
\hline Mar. 2020 & 17773 & User Knowledge Q\&A & 32 \\
\hline Apr. 2020 & 12132 & Public Knowledge Q\&A & 22 \\
\hline May. 2020 & 20586 & System Skills & 7 \\
\hline Jun. 2020 & 12047 & Default Replies & 2 \\
\hline
\end{tabular}

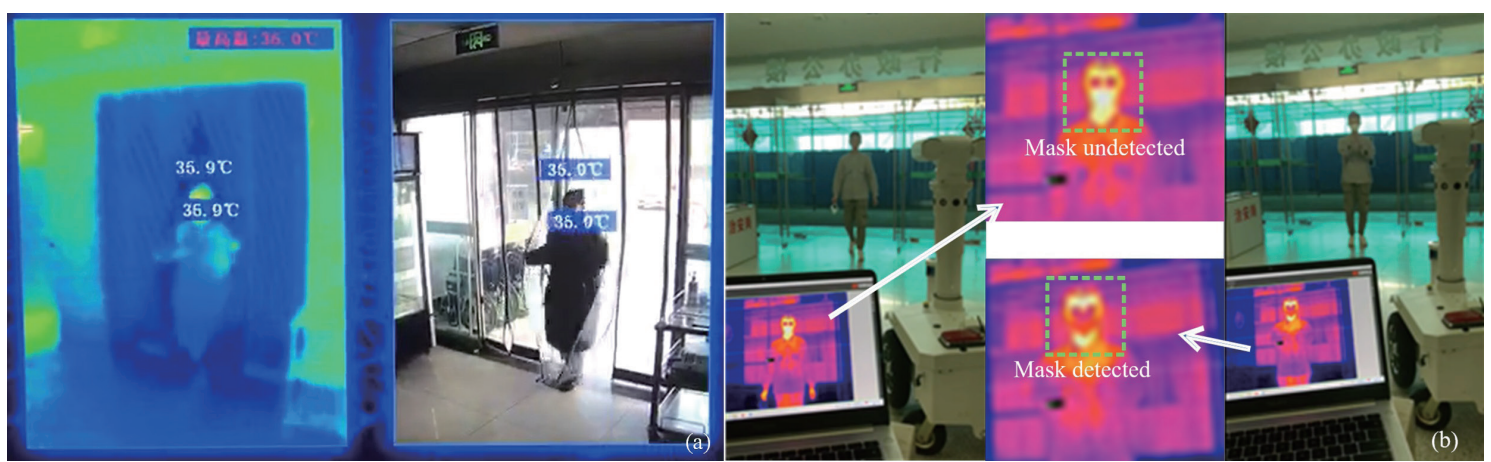

Fig. 8. (Color online) (a) IR continuous body temperature measurement, during which the maximum temperature is extracted. (b) Comparison of a masked face and an unmasked face.

Table 3

Statistics of temperature detection at several hospitals using the proposed system.

\begin{tabular}{lcrrrr}
\hline & & \multicolumn{3}{c}{ Month } & \multirow{2}{*}{ Total } \\
\cline { 3 - 5 } & & April & May & June & \\
\hline \multirow{2}{*}{ Beijing } & \# of people & 3698 & 410131 & 362061 & 775890 \\
& $\geq 37.2^{\circ} \mathrm{C}$ & 782 & 32547 & 47461 & 80790 \\
\hline Inner & $\#$ of people & 9634 & - & - & 9634 \\
Mongolia & $\geq 37.2^{\circ} \mathrm{C}$ & 43 & - & - & 43 \\
\hline \multirow{2}{*}{ Anhui } & $\#$ of people & 614 & 1039 & 7678 & 9325 \\
& $\geq 37.2^{\circ} \mathrm{C}$ & 23 & 60 & 216 & 299 \\
\hline
\end{tabular}




\section{Discussion and Conclusions}

In this article, we proposed a series of service robots with multiple sensors under cloud control based on the analysis of the specific application environment and the requirements of the Wuhan cabin hospitals. The details of the system architecture and several applications were presented and developed, such as autonomous disinfection, delivery, cleaning, temperature measurement, and natural communication. The framework can quickly and flexibly support independent robot functional applications according to the application conditions, and can also be expanded to multi-person-multi-robot collaborative work assisted by the sensors used by the service robots, $5 \mathrm{G}$, and cloud and artificial intelligence technologies in a large working area. However, owing to the short duration of the cabin hospitals, a large part of this study is still in its conception. In the future, several multi-person-multi-robot collaborative work application scenarios could be further considered, such as the following: (1) Collaborative work by multiple robots of the same type. For example, disinfection robots may work in coordination to perform spraying and disinfecting tasks, where the work area may be distributed among the robots for efficiency and efficacy. (2) Collaborative work by multiple robots of different types. For example, a temperature-measuring robot may work with a reception robot. When the temperature-measuring robot detects that a patient has a fever, the cloud will command the nearest reception robot to ask questions and answer the questions from patients, understand the patient's current state, and calm the patient. Based on the proposed framework, these concepts could be implemented by task scheduling in the application management module according to specific application requirements. With the development of new sensors, we will continue to improve the proposed cloud-based human-centered multi-robot collaboration architecture, track and observe the global development of the COVID-19 epidemic, and develop and deploy optimal applications according to the progress of the epidemic. We hope that our work will inspire other research groups.

\section{References}

1 W. H. Organization: Report of the WHO China Joint Mission on Coronavirus Disease 2019 (COVID-19) (2020). http://asean-china-center.org/resources/file/P020200318828172605354.pdf (accessed Feb. 2020).

2 M. Roser, H. Ritchie, E. Ortiz-Ospina, and J. Hasell: Coronavirus Disease (COVID-19) - Statistics and Research, Our World in Data (2020). https://ourworldindata.org/coronavirus

3 J. Hellewell, S. Abbott, A. Gimma, N. I. Bosse, C. I. Jarvis, T. W. Russell, J. D. Munday, A. J. Kucharski, W. J. Edmunds, F. Sun, S. Flasche, B. J. Quilty, N. Davies, Y. Liu, S. Clifford, P. Klepac, M. Jit, C. Diamond, H. Gibbs, K. V. Zandvoort, S. Funk, and R. M. Eggo: Lancet Global Health 8 (2020) 488496. https://doi. org/10.1016/S2214-109X(20)30074-7

4 W.-Y. G. Louie, J. Li, T. Vaquero, and G. Nejat: Proc. IEEE Int. Symp. Robot and Human Interactive Communication (IEEE, 2014) 237-242. https://doi.org/10.1109/ROMAN.2014.6926259

5 G.-Z. Yang, B. J. Nelson, R. R. Murphy, H. Choset, H. Christensen, S. H. Collins, P. Dario, K. Goldberg, K. Ikuta, N. Jacobstein, D. Kragic, R. H. Taylor, and M. Mcnutt: Sci. Rob. 5 (2021) 1. https://doi.org/10.1126/ scirobotics.abb5589

6 A.-Z. Gao, R. R. Murphy, W. Chen, G. Dagnino, P. Fischer, M. G. Gutierrez, D. Kundrat, B. J. Nelson, N. Shasudhin, H. Su, J. Xia, A. Zemmar, D.-D. Zhang, C. Wang, and G.-Z. Yang: Sci. Rob. 6 (2021). https://doi. org/10.1126/scirobotics.abf 1462

7 A. G. Ozkil, Z. Fan, S. Dawids, H. Aanaes, J. K. Kristensen, and K. H. Christensen: 2009 Proc. IEEE Int. Conf. Automation and Logistics (2009) 289-294. https://doi.org/10.1109/ical.2009.5262912 
8 M. Podpora, A. Gardecki, and A. Kawala-Sterniuk: IFAC-PapersOnLine 52 (2019) 347. https://doi.org/10.1016/j. ifacol.2019.12.685

9 E. J. Hwang, B. K. Ahn, B. A. Macdonald, and H. S. Ahn: 2020 IEEE Int. Conf. Robotics and Automation (ICRA2020) 8013-8018. https://doi.org/10.1109/ICRA40945.2020.9197160

10 C. R. Kovach, Y. Taneli, T. Neiman, E. M. Dyer, A. J. A. Arzaga, and S. T. Kelber: BMC Infect. Dis. 17 (2017) 186. https://doi.org/10.1186/s12879-017-2275-2

11 P. Courtney and P. G. Royall: IEEE Rob. Autom. Mag. 28 (2021) 28. https://doi.org/10.1109/MRA.2020.3045067

12 C. Tamantini, F. Scotto di Luzio, F. Cordella, and G. Pascarella, L. Zollo: IEEE Rob. Autom. Mag. 28 (2021) 71. https://doi.org/10.1109/MRA.2020.3044953

13 A. D. Lallo, R. Murphy, A. Krieger, J. Zhu, and H. Su: IEEE Rob. Autom. Mag. 28 (2021) 18. https://doi. org/10.1109/MRA.2020.3045671.

14 M. Bonaccorsi, L. Fiorini, F. Cavallo, A. Saffiotti, and P. Dario: Int. J. Soc. Robot 8 (2016) 393. https://doi. org/10.1007/s12369-016-0351-1

15 S. Reig, M. Luria, J. Z. Wang, D. Oltman, E. J. Carter, A. Steinfeld, J. Forlizzi, and J. Zimmerman: Proc. ACM/ IEEE Int. Conf. Human-robot Interaction (ACM/IEEE 2020) 289-297. https://doi.org/10.1145/3319502.3374795

16 X. S. Zhou and S. I. Roumeliotis: Proc. IEEE/RSJ Int. Conf. Intelligent Robots and Systems (IEEE/RSJ 2006) 1785-1792. https://doi.org/10.1109/iros.2006.282219

17 S. Saeedi, M. Trentini, M. Seto, and H. Li: J. Field Rob. 33 (2016) 3. https://doi.org/10.1002/rob.21620

18 B. K. Patle, G. Babu L, A. Pandey, D. R. K. Parhi, and A. Jagadeesh: Defence Technol. 15 (2019) 582. https:// doi.org/10.1016/j.dt.2019.04.011

19 H. Durrant-Whyte and T. Bailey: IEEE Rob. Autom. Mag. 13 (2016) 99-110. https://doi.org/10.1109/ MRA.2006.1638022

20 R. Mur-Artal, J. M. M. Montiel, and J. D. Tardós: IEEE Trans. Rob. 31 (2015) 1147. https://doi.org/10.1109/ TRO.2015.2463671

21 D.-P. Zou, P. Tan, and W.-X. Yu: Virtual Real. Intell. Hardware I (2019) 461. https://doi.org/10.1016/j. vrih.2019.09.002

22 L. Riazuelo, J. Civera, and J. M. M. Montiel: Rob. Auton. Syst. 62 (2014) 401. https://doi.org/10.1016/j. robot.2013.11.007

23 D. Peña and F. Tanaka: 2018 ACM/IEEE Int. Conf. Human-robot Interaction. (ACM/IEEE, 2018) 207-208.

24 V. W. Zue: Proc. IEEE 73 (1985) 1602. https://doi.org/10.1109/PROC.1985.13342

25 M. Wolfel: 2008 IEEE Int. Conf. Acoustics, Speech and Signal Processing (IEEE, 2008) 4705-4708. https:// doi.org/10.1109/ICASSP.2008.4518707

26 Z. Wang and D. Wang: IEEE/ACM Trans. Audio Speech Lang. Process. 24 (2016) 796. https://doi.org/10.1109/ TASLP.2016.2528171

27 L. A. Zadeh: IEEE Trans. Syst. Man Cybern. SMC-3 (1973) 28. https://doi.org/10.1109/TSMC.1973.5408575

28 M. T. Mills and N. G. Bourbakis: IEEE Trans. Syst. Man Cybern.: Syst. 44 (2014) 59. https://doi.org/10.1109/ TSMCC.2012.2227472

29 A. Agarwal, C. Baechle, R. Behara, and X. Zhu: IEEE J. Biomed. Health Inf. 22 (2018) 588. https://doi. org/10.1109/JBHI.2017.2684121

30 G. Dimauro, V. Di Nicola, V. Bevilacqua, D. Caivano, and F. Girardi: IEEE Access 5 (2017) 22199. https://doi. org/10.1109/ACCESS.2017.2762475

31 C.-Y. Yeh and S.-H. Hwang: IEEE Proc. - Vision, Image and Signal Processing 152 (2005) 793-799. https://doi. org/10.1049/ip-vis:20045095

32 A. Gibiansky, S. Arik, G. Diamos, J. Miller, K. Peng, W. Ping, J. Raiman, and Y. Zhou: Proc. Advances in Neural Information Processing Systems (2017) 2962-2970.

33 S. Machida, S. Shishido, T. Tokuhara, M. Yanagida, T. Yamada, M. Izuchi, Y. Sato, Y. Miyake, M. Nakata, M. Murakami, M. Harada, and Y. Inoue: Solid-State Circuits 53 (2018) 229. https://doi.org/10.1109/ JSSC.2017.2769341.

34 H. Jiang and E. Learned-Miller: Proc. 2017 IEEE Int. Conf. Automatic Face \& Gesture Recognition (FG 2017), 650-657. https://doi.org/10.1109/FG.2017.82

35 Ç. Şenaras, B. Yüksel, M. Özay, and F. Yarman-Vural: Proc. 2012 IEEE Int. Geoscience and Remote Sensing Symp. (IEEE, 2012) 6713-6716. https://doi.org/10.1109/igarss.2012.6352058 


\section{About the Authors}

Sheng Bao received her bachelor's (1998), master's (2000), and Ph.D. (2004) degrees from Harbin Institute of Technology, China. She worked as postdoctoral researcher (2006) at Tsinghua University and as an assistant professor (2009) at Shanghai Robotics Institute, Shanghai University. She was a visiting scholar of Vanderbilt University in 2014. Her current research interests include kinematic and dynamic algorithms of manipulators and joint servo driving and control. (baos@shu.edu.cn)

Bill Huang received his B.S. degree from Huazhong University of Science and Technology, China, in 1982. He worked at Bell Laboratories in the United States and was a senior vice president and chief technology officer of UT Starcom until 2006. He was president of China Mobile Communications Research Institute in 2007. He is the founder of CloudMinds Technologies Inc. and has been its CEO since 2015. He is committed to developing cloud intelligent robot technology. (bill@cloudminds.com)

Jianjun Yuan has been working as a professor and as deputy director of Shanghai Robotics Institute, Shanghai University, since 2019. He received his bachelor's (1999) and master's (2001) degrees from Tsinghua University, China, and his Ph.D. (2005) degree from Tokyo Institute of Technology, Japan. He worked as a postdoctoral researcher (2006) at Tokyo Institute of Technology and as an associate professor (2006-2019) at the Research Institute of Robotics, Shanghai Jiao Tong University. He was a visiting scholar of CMU in 2014 and a Pu Jiang Scholar of Shanghai City in 2007. His research interests include innovative design, manipulators and applications, kinematic and dynamic algorithms, and special-needs robotics. He is a senior member of IEEE. (terabit@shu.edu.cn)

Bing Wang received his MBA degree from the University of Hertfordshire, UK, in 2010. Since graduating in 1988, he has been engaged in network security research and is a leader of a team creating a new security mechanism used in a variety of software systems. He is currently building a security system applied to cloud robots. (charly@cloudminds.com)

Lin Xia received his B.S. degree from Shanghai Jiao Tong University in 1998. Since his first project, he has focused on telecoms and software platforms. In 2016, he joined an AI team that applies AI technology to life services. His main research directions are better human-robot interaction and smarter AI based on cloud robot solutions. (linairy@163.com)

Mingyuan Wang received his B.S. and M.S. degrees from Shanghai University of Engineering Science, China, in 2017 and 2020, respectively. He is currently working toward his Ph.D. degree at Shanghai Robotics Institute, Shanghai Key Laboratory of Intelligent Manufacturing and Robotics, Shanghai University. His research interests are in mechanical design and teleoperation robotics. (wmy20008@163.com) 
Yanchen Liu received her B.S. degree from Xidian University in 2014. From 2015 to 2018, she worked as a communication engineer, designing a communication system used between ships and land command centers. Since 2019, she has been researching communication systems used by cloud robots. (Vicky.liu@cloudminds.com) 